This item was submitted to Loughborough's Research Repository by the author.

Items in Figshare are protected by copyright, with all rights reserved, unless otherwise indicated.

\title{
Promoting exercise behaviour in a secure mental health setting: health care assistant perspectives
}

\section{PLEASE CITE THE PUBLISHED VERSION}

https://doi.org/10.1111/inm.12484

\section{PUBLISHER}

(c) Australian College of Mental Health Nurses. Published by Wiley

\section{VERSION}

AM (Accepted Manuscript)

\section{PUBLISHER STATEMENT}

This work is made available according to the conditions of the Creative Commons Attribution-NonCommercialNoDerivatives 4.0 International (CC BY-NC-ND 4.0) licence. Full details of this licence are available at: https://creativecommons.org/licenses/by-nc-nd/4.0/

\section{LICENCE}

CC BY-NC-ND 4.0

\section{REPOSITORY RECORD}

Kinnafick, Florence-Emilie, Anthony Papathomas, and Dora Regoczi. 2019. "Promoting Exercise Behaviour in a Secure Mental Health Setting: Health Care Assistant Perspectives”. figshare. https://hdl.handle.net/2134/33027. 
Manuscript category: Original article

\section{Promoting exercise behaviour in a secure mental health setting: Health Care Assistant perspectives}

Kinnafick, Florence Emilie. ${ }^{1}$, Papathomas, Anthony. ${ }^{1}$, Regoczi, Dora ${ }^{1}$.

${ }^{1}$ National Centre for Sport and Exercise Medicine, School of Sport, Exercise and Health Sciences, University of Loughborough, LE11 3TU, UK

Authorship statement: Dora Regoczi conducted all interviews and transcribed the data (Substantial contributions to the acquisition). Anthony Papathomas analysed the data and wrote the methods section (Substantial contributions to the design of the work, analysis, and interpretation of data; Drafted and revised the work critically for important intellectual content; provided final approval of the version to be published. Florence Kinnafick was project lead, analysed the data and wrote the introduction, results, discussion and conclusions (Substantial contributions to the conception, design of the work; analysis, and interpretation of data for the work; and revised the work critically for important intellectual content; provided final approval of the version to be published.

*Corresponding author: F-E. Kinnafick (email: f.e.kinnafick@lboro.ac.uk)

${ }^{1}$ Room 1.24. National Centre for Sport and Exercise Medicine, School of Sport, Exercise and Health Sciences, Loughborough University, LE11 3TU, UK

Phone number: 01509226364

F. E. Kinnafick ORCID 0000-0002-3095-7116

A Papathomas ORCID 0000-0002-1031-9256

Acknowledgements: We would like to thank St Andrews Healthcare Charity for providing access to this unique setting and aiding the process of recruiting HCAs to take part in the interviews.

Declaration of interest: The author's declare that they have no conflict of interest

Word count: 4440 


\title{
Promoting exercise behaviour in a secure mental health setting: Health Care Assistant perspectives
}

\begin{abstract}
Individuals with severe mental illness engage in significantly less amounts of physical activity than the general population. A secure mental health setting can exacerbate barriers to exercise, and facilitate physical inactivity and sedentary behaviour. Health Care Assistants are intimately involved in the daily lives of patients and therefore, should be considered integral to exercise promotion in secure mental health settings. Our aim was to explore Health Care Assistants perceptions of exercise and their attitudes to exercise promotion for adult patients in a secure mental health hospital. Qualitative semi-structured interviews were conducted with 11 Health Care Assistants from a large UK based secure mental health hospital. Topics included Health Care Assistants personal experiences of exercise within a secure facility, their perceptions of exercise as an effective treatment tool for mental health, and their perceived roles and responsibilities for exercise promotion. Thematic analysis was used to analyse the data. Three main themes were identified; 1) exercise as multiply beneficial for patients, 2) perceived barriers to effective exercise promotion, and 3) strategies for effectives exercise promotion. Health Care Assistants considered exercise to hold patient benefits. However, core organisational and individual barriers limited Health Care Assistants exercise promotion efforts. An informal approach to exercise promotion was deemed most effective to some, whereas others committed to more formal strategies including compulsory sessions. With education and organisational support, we propose Health Care Assistants are well placed to identify individual needs for exercise promotion. Their consultation could lead to more efficacious, person-sensitive interventions.
\end{abstract}

Key words: exercise promotion, qualitative, secure setting, severe mental illness, 


\section{EXERCISE IN A SECURE MENTAL HEALTH SETTING}

\section{Introduction}

Individuals with severe mental illness (SMI) experience premature mortality of up to 20 years (Walker et al. 2015). This mortality rate is largely linked to physical health inequalities which can be ameliorated through modifiable risk factors such as diet and exercise (Ribe et al. 2014). A healthy lifestyle is particularly relevant for those receiving antipsychotic medication which, when combined with large amounts of sedentary behaviour, can significantly increase cardio-metabolic risk (Vancampfort et al. 2016).

\section{Background}

A growing body of work supports the benefits of exercise for physical and mental health for people with SMI in inpatient settings (Stanton \& Happell 2014). However, people with SMI continue to engage in significantly less exercise and more sedentary behaviour compared to the general population (Schuch et al. 2017). The restrictive environment of a secure setting, where patients are not freely able to move between wards or venture outside without an appropriate escort, can exacerbate barriers to exercise, and facilitate physical inactivity and sedentary behaviour (Robertson et al. 2000). Secure wards are populated by patients with complex, individual health needs in an environment with high staff turnover and high staffing demands (Cleary et al. 2011).

Public Health England's recent report on reducing levels of obesity in secure settings emphasised the importance of ward staff in supporting lifestyle change interventions (Day \& Johnson 2017). Although previous work has explored the attitudes to exercise promotion of mental health nurses (e.g., Faulkner \& Biddle 2002; Happell et al. 2012), the perspectives of health care assistants (HCAs) are undocumented. Yet HCAs are more intimately involved in the daily lives of patients than mental health nurses; presenting on wards 24 hours per day, delivering a third of the caring workforce and spending more time providing hands-on care (Cavendish 2013). As such, HCAs should be considered integral to exercise promotion in secure mental health settings. However, HCAs have typically received less formal education and training than mental health nurses (Cavendish 2013). Although the recently proposed 'Certificate of Fundamental Care’ seeks to provide baseline knowledge requirements for care workers; it is only 


\section{EXERCISE IN A SECURE MENTAL HEALTH SETTING}

compulsory for newly appointed HCAs and none of its 15 'core standards' address physical activity or exercise (Cavendish 2013). In light of the above, the aim of this research is to explore HCA perceptions of exercise and attitudes to its promotion for adult patients in a secure mental health hospital.

\section{Methods}

In accordance with the ethical standards laid down in the 1964 Declaration of Helsinki and its later amendments, the current study was approved by the University Ethics (Human Participants) Committee. Following ethical approval qualitative semi-structured interviews were conducted with 11 HCAs who worked in a large UK based secure mental health hospital.

\section{Participants}

Participants were included if they had worked in the institution for a minimum of 6 months to ensure they were familiar with the workings of the hospital. The 11 (5 male, 6 female) participants were aged between 22 and 48 years old $(M=30.27, S D=7.75)$. Work experience at the hospital ranged from 6 months to 18 years $(M=5.45, S D=5.04)$. Participants were recruited from 7 hospital wards including female and male adolescent, women's mental health, male with mainly autism spectrum disorder, learning disability, forensic, and also women aged above 55 years with diverse mental illness.

\section{Data collection}

An opportunistic approach to recruitment was used whereby the researcher visited the wards with another member of staff to offer information about the study. HCAs were given the opportunity to ask questions before providing written informed consent. The accompanying member of staff then replaced the HCA on the ward to ensure the ward was suitably staffed. Interviews took place in a private room where the HCAs could speak freely. Interviews were recorded using a digital voice recorder and ranged between 20 to 60 minutes $(M=40.16, S D=12.7)$. Approximately 9 hours of data was collected.

The interview schedule was created to gather information focusing on the uniqueness of each HCAs perceptions, beliefs, attitudes and general experiences of exercise and exercise promotion. Open ended questions facilitated discussion and provided flexibility during the 


\section{EXERCISE IN A SECURE MENTAL HEALTH SETTING}

interviews. They included practical questions (e.g., 'Please describe your personal view of exercise and mental health?'), theoretical questions (e.g., 'How do your patients exercise? What are your thought of exercise as an option for your patients?'), and guiding questions (e.g., ‘Can you tell me about a time where you tried to promote exercise?').

\section{Data analysis}

This research adhered to an interpretive paradigm whereby the focus was subjective experience not objective reality. Thematic analysis of data was guided by the rigorous methodological steps described by Braun and Clarke (2006). This systematic process for identifying patterns across transcripts includes the following: repeated readings of the transcripts, assigning codes (content summarising labels) to data deemed relevant to our research question, grouping similar codes into broader themes, before naming these broader conceptual themes and writing-up the results.

\section{Results}

Three main themes were identified through our analysis; 1) exercise as multiply beneficial for patients, 2) perceived barriers to effective exercise promotion, and 3) strategies for effectives exercise promotion. The main themes with corresponding subthemes are detailed in Table 1.

\section{[INSERT TABLE I HERE]}

\section{Theme 1: Exercise as multiply beneficial to patients}

All HCAs agreed that exercise was beneficial for patients’ physical and mental health. Positivity towards exercise was underpinned by two subthemes; a 'normalising' effect and a therapeutic tool.

A 'normalising' effect. Exercise was judged an opportunity to provide patients with respite from the daily routines that characterize secure mental health care:

It is really beneficial. Something as simple as just getting out for a walk on the grounds, it can just refresh your entire view of the day. It gives you some time away from the ward...these patients don't want to sit in 24 hours, they want to get out. [Participant 9].

Exercise was considered a way for patients to connect with a world beyond mental ill health and care:

Exercise gives them an option to forget that they got that diagnosis, they are just teenage lads playing football or taking part in boxing classes. I think that is a really good thing. Exercise will always be there in their entire life as a way to get away from their diagnosis [Participant 9] 
A therapeutic tool. HCAs expressed conflicting views on the effectiveness of exercise as a therapeutic tool. Although perceived as beneficial, some HCAs were reluctant to call exercise a therapy in its own right or consider it a formal part of the treatment process:

It definitely has therapeutic benefits. It's not a therapy in itself. I think it's not fixing anything, or solving a problem it's just helping manage it and it's more like a tool, I guess [Participant 4].

Exercise was also deemed to hold a calming effect, reducing emotional distress to a level whereby the patient is receptive to traditional support:

When someone is agitated... experiencing a lot of emotional distress. One of the main sorts of thing for that is to bring them back down with intense exercise...that's why we got a gym on the ward. When people are feeling distressed they go on the cross-trainer, really go for it for 5 minutes and bring their level of distress down. It seems to have a massive effect on people. It helps them sort of come down to a level where they can talk about what the problem is...maybe come up with a solution [Participant 3].

On the other hand, HCAs expressed concerns that the symptoms of some mental illnesses might lead to unhealthy exercise practices:

There can be negative effects as well. A couple of guys, especially one patient who would just exercise on a machine, non-stop and needs to be prompted to stop after an hour of doing circuits. With his sort of psychology...that's another issue as well I suppose, body image and going too far [Participant 5].

\section{Theme 2: Perceived barriers to effective exercise promotion}

HCAs discussed a range of barriers that limited exercise promotion efforts. These perceived barriers were characterized by two sub-themes; organisational culture in a secure setting and inconsistent ‘buy-in’ from staff.

Organisational culture. HCAs described how various organisational cultural issues associated with secure mental health care prevented effective and consistent exercise promotion. HCAs highlighted that because exercise is not part of a patient's personalised care plan, priority was afforded to medication and traditional psychotherapies:

When it comes to discussing patients' care plans and stuff like that it always comes down to their medication and their psychological well-being because that is the two main things, that's why they are here, I guess [Participant 3].

HCAs also proposed that inadequate staffing could limit their capacity to promote exercise: 
If one of the guys wants to go out to use the outdoor gym but we don't have enough staff, that can stop them from going, which is not a good thing. You don't want to get to the point when they just give up asking because there is not enough staff so kind of what's the point, I can't get out so I can't do. So there can be a negative outcome [Participant 6].

One HCA argued that staffing allocations did not account for the fact exercise sessions must be fully supervised:

The only problem is sometimes if there are sessions off the ward, if there are issues with staffing or obviously a situation is going on in the ward that is taking staff away who would be able to escort; that could be a bit of a problem. I don't think that always gets considered. Staffing numbers don't reflect making sure that the sessions like these that are really important, like the exercise ones, always happen. In the past, some patients did miss out on those sessions because of staffing levels [Participant 3].

Although HCAs recognised the critical importance of patient safeguarding procedures, they also identified these as presenting a barrier to their exercise promotion efforts:

There might be the guys who have restricted leave so sometimes you are physically unable to facilitate certain sessions because they are not allowed to do certain things. Safeguarding issues; if it's a group activity, whether there is another person who has a conflict with the guy you want to take there. They can't be there at the same time [Participant 6].

One HCA identified a tension between effective and responsible patient safeguarding and the potential benefits of exercise to patients:

You've got to assess the patient. If you want to take them out but you can see that they are punching walls and they are saying 'take me out because that's going to burn my energy'. If their mental state is not looking great, you should not let them out even if that's what they need, if they are not safe, they should not go. There are boundaries [Participant 9].

Concerns over HCA safeguarding were considered an additional barrier to exercise promotion in secure settings:

There are a lot of patients if you let them out with an inexperienced staff member, they can run. With the gym...heavy objects and they are having a bad mood, they could hurt you [Participant 9].

Inconsistent staff “buy-in”. HCAs agreed that promoting patient wellbeing was a chief responsibility of the role, but the extent to which they should encourage exercise was unclear to them. Even when staff bought-in to the idea of exercise promotion, competency doubts and conflicting beliefs limited this:

I see my role as therapeutic engagement. I try to help out with whatever they are struggling with. It can be their mental health, family issue, or for example when a patient approached me and said 'I've had enough, I want to lose weight'. So I'm taking her to the gym regularly. I think our job is to promote pretty much everything; we are not here to sit and babysit them 
[patients]. But it depends on the person as well. I really like exercise, but I'm not sure about advising them. It is hard, because I think it is our job to be therapeutic but then I know that it is not really my job role like, to make sure that that person exercises. [Participant 2]

Some HCAs however, acknowledged that although exercise promotion was not specifically outlined in their job description, they were committed to promote any activity that had the potential to improve the wellbeing of patients:

It is not specifically outlined within the role but I think in a nursing team, it just becomes very evident when you start work here that part of your role is to encourage people to do the effective things that are going to be beneficial to them on a therapeutic level. Exercise is one of them. I think it's always been something that we try to encourage as much as we can. It is just whether or not we can. It is whether or not we have the resources in terms of staff and make sure that it happens [Participant 3].

\section{Theme 3: Strategies for successful behaviour change}

HCAs communicated contrasting beliefs regarding how best to get patients active. These were separated by two sub-themes; Informal strategies and compulsory intervention. Whereas some proposed informal strategies to exercise promotion, others believed a more proactive and compulsory intervention was required.

Informal strategies. A key part of understanding what strategies could be used for promoting successful exercise behaviour change was the importance of building rapport and understanding patient needs:

It is getting to know your patients and finding out what they are interested in, not what you are interested in, what they are interested in. Trying to get them excited, to see what they want to do...so having a positive relationship, it is extremely hard and definitely helps. Some days it is even hard to get them out from their bed. So it is really challenging [Participant 2]

Informal processes also included providing informational and social support to the patients:

You could promote in loads of different ways. You can just talk with them, put up posters about stuff that is going on. In their sessions, community meetings tell them what kind of stuff is going on that next week. Chat with them, you are with them every day. You can talk about exercise whenever. And if you hear something, just pass on the message. You are talking to them anyway [Participant 9].

Those HCAs who had used, or discussed, informal strategies typically viewed exercise as the individual responsibility of the patient: 
We can only encourage them. We all try to go around every morning but if they have nothing to get up for, they are not going to. And no matter how exciting you make something sound, if they don't want it, they won't [Participant 11].

Some HCAs believed patients were more receptive to exercise if they were autonomous in their decision to engage with it and more likely to resist if they felt pressured into it:

We don't tend to push the ladies to do it, they tend to do it on their own. Once they choose to do it on their own, it is their decision. If we push them more to do it, they just not going to do it [Participant 10].

HCAs acknowledged they were in a position to create an environment where patients were more

likely to take up opportunities to exercise:

It is in the control of the patient primarily but we have control of giving them the opportunities to expose them to exercise in different ways. Not to limit their opportunities with exercise but to give them the option to take it upon themselves whenever they want. You can never know when they're going to be in a mind state to try something new, something else. So you need to be ready to provide it [Participant 4].

Compulsory intervention. In contrast, some HCAs suggested compulsory sessions would ensure patients could benefit from the outcomes of exercise:

The girls get exercise sessions on their timetable most days. Most days if not all days. But they are not compulsory, they are optional which I think needs changing to be honest. I think it should be compulsory because all the other things are. When a patient has a compulsory session, if they don't attend their session, means that they are not allowed to do what they need. So if they want to go to the shops, they have to attend all of their session to be able to do that [Participant 7].

Not all HCAs agreed that this strategy for exercise promotion would be successful and, in fact, could be detrimental to the patients:

A treatment option where everybody has to do it just wouldn't work. That just sends you back to school times, and probably be quite negative for a lot of people. But provided as an option it's something which will be taken advantage of and promoted for people to try [Participant 4].

\section{Discussion}

This original study is the first to explore health care assistants’ perspectives on exercise promotion within the unique context of a secure mental health service. All HCAs considered exercise to hold patient benefits with some HCAs suggesting it could fulfil an important therapeutic role. Irrespective of this positivity, core organisational and individual barriers limited HCAs exercise promotion efforts. 


\section{EXERCISE IN A SECURE MENTAL HEALTH SETTING}

On an organisational level, regimented safeguarding procedures limited exercise opportunities. On an individual level, HCAs held conflicting views as to whether or not exercise promotion was their responsibility. For those HCAs that did see exercise promotion as a job role, there were discrepant opinions on how best to achieve this. Whereas an informal approach to exercise promotion was deemed most effective to some, others committed to more formal strategies including compulsory sessions. In the ensuing paragraphs we interpret these findings in light of existing research and implications for policy and practice.

HCAs’ positivity towards exercise in secure mental health settings echoes the perspectives of mental health nurses in non-secure mental health services (e.g., Faulkner \& Biddle 2002; Happell et al. 2012). The assertion that exercise-related activities may provide patients with a "normalising" experience is a plausible hypothesis worthy of further investigation. Previous findings have associated exercise with feelings of distraction (Cullen \& Mccann 2015) and a move away from an illness identity (Carless \& Douglas 2008) but it is not known whether these benefits also occur within the confines of a secure mental health service. Exercise was also seen as presenting direct treatment benefits for mental illness but this was a less pervasive view and there was overt disagreement among the sample. Difficulty accepting physical solutions to mental health problems is present across a range of professionals working in psychiatric services (e.g., Faulkner \& Biddle 2002; Faulkner \& Biddle 2001). This may be a consequence of disciplinary (i.e., psychiatry and psychology) subscription to Cartesian dualism and the idea that the mind and body are separate entities with little interaction (Phillips et al. 2014). For HCAs, who are not required to receive formal disciplinary education, subscription to mind-body dualism may be a reflection of how senior practitioners shape the organisational culture HCAs inhabit. Understanding how senior psychiatrists conceive the role of exercise as a treatment for SMI in secure settings is an important future research direction.

Despite an overwhelming body of evidence supporting the role of exercise as essential in the treatment of mental illness (Western et al. 2014; Day \& Johnson 2017), the experiences of HCAs in this study suggest exercise was rarely properly integrated into mental health care and not consistently being applied in secure settings (Faulkner \& Biddle 2002). HCAs did not view exercise sessions as a priority because they were not integrated as part of an official care plan in the same way as 


\section{EXERCISE IN A SECURE MENTAL HEALTH SETTING}

medication or other therapies (e.g., counselling). Additional organisational barriers, associated with the restrictive nature of the secure setting, also contributed to inconsistent exercise promotion (e.g., low staffing levels, restricted leave, safeguarding issues). Secure services should therefore consider ways to improve staff provision specifically for the needs of exercise sessions. When staff levels are low, HCAs should be encouraged to facilitate physical activity or encourage reduced sedentary time within the wards to contribute to the health benefits of moving more (Stanton \& Happell 2014).

Existing research has partly attributed the lack of exercise in patients with SMI to the attitude towards health promotion of those who deliver an intervention (Suija et al. 2009). For example, if the practitioner is sceptical about exercise or their role in promoting the behaviour, patient engagement in the behaviour is limited (Suija et al. 2009). Although all HCAs described a responsibility to promote patient wellbeing; only a few connected exercise to this remit and fewer still felt sufficiently qualified to promote exercise effectively. Literature has identified a lack of training for healthcare professionals in encouraging highly sedentary patients with mental illness to become more physically active (Crone et al. 2008) and Public Health England's recent review highlights that organisations should train staff in health improvement specifying the inclusion of exercise and exercise promotion (Day \& Johnson 2017). Our findings are aligned to earlier work that concluded mental health nurses are well positioned to encourage and assist patients to engage in exercise, although they might lack the educational preparation to perform this role effectively (Happell et al. 2011). We suggest that for HCAs, who are now spending more time interacting with patients, further knowledge of exercise promotion and role clarification/specification would be welcomed to enhance exercise promotion.

Framing HCAs’ perceptions according to the psychology of behaviour change, the informal strategies suggested might facilitate individual volition; a core feature of sustained engagement with exercise (Ng et al. 2012). In contrast, the controlling nature of a compulsory intervention is thought to undermine the autonomous motivation required for long term exercise behaviour and improved wellbeing (Ng et al. 2012). Research underpinned by theories of motivation (e.g., self-determination theory) have shown that those who are extrinsically motivated to exercise (e.g., instructed to by a health professional) are less likely to continue with the behaviour once the extrinsic motivator has ceased (Deci \& Ryan Richard 2008). Although individuals with SMI have demonstrated motivational 


\section{EXERCISE IN A SECURE MENTAL HEALTH SETTING}

and volitional deficits when explaining exercise participation compared to a non-depressed control (Kramer et al. 2014), literature supports that it is a good quality of motivation to exercise (i.e., autonomous motivation) that can influence exercise behaviour for individuals with SMI (Vancampfort et al. 2013). Future work should explore the most effective exercise promotion strategies for autonomous exercise engagement for long lasting behaviour change. Furthermore, instigating obligatory exercise serves to endorse it as a "treatment” which may negate many of the added benefits identified by HCAs (i.e. normalising activity, escape from routine) although would ensure that patients do benefit from both the physiological and psychological benefits of exercise (Western et al. 2014). This points to a potential paradox whereby if exercise is deemed effective it will likely become integrated into routine care, yet if it becomes routine care it may cease to be effective. Secure mental health services must resolve this tension by ensuring exercise is regular enough to be physically and mentally beneficial but not so structured that its inherent pleasure is nullified.

\section{Strengths and limitations}

Although our original findings contribute to existing literature (Faulkner \& Biddle 2002; Farholm \& Sørensen 2016), there are some limitations to consider. This study was conducted in a low and medium secure setting within a large hospital, therefore future research should consider staff perceptions across other healthcare settings (e.g., smaller hospitals and/or high secure units). Interviews took place on site with staff across 7 different wards from varying care pathways. This provided a broader view of HCA perceptions and attitudes to exercise promotion for patients with differing needs. Despite the efforts we made as researchers to create a trusting and non-judgmental environment, it is possible that the participants responded with social desirability during interviews because the interviews were conducted in their place of work. It is also possible that HCAs with an interest in exercise were more likely to volunteer to participate in the focus groups. A particular strength of the study is the focus on HCA staff to explore perceptions of health promotion. HCAs now make up over one third of the caring workforce in hospitals and spend more time with patients than registered nurses although may have received much less, and inconsistent training (Cavendish 2013).

\section{Conclusion and relevance for clinical practice}




\section{EXERCISE IN A SECURE MENTAL HEALTH SETTING}

We aimed to explore health care assistants' perspectives on exercise promotion within the unique context of a secure mental health service. HCAs considered exercise to hold patient benefits. However, core organisational and individual barriers limited HCAs exercise promotion efforts. An informal approach to exercise promotion was deemed most effective to some, whereas others committed to more formal strategies including compulsory sessions. By including the perspectives of the HCA adds to extant information with the aim of developing more effective strategies in this unique setting. We propose that HCAs are best placed to identify individual patient needs for exercise promotion. With appropriate education and organisational support their consultation in the implementation of exercise promotion strategies could lead to more ethical forms of exercise promotion with more efficacious, person-sensitive interventions (Williams et al. 2017). 


\section{EXERCISE IN A SECURE MENTAL HEALTH SETTING}

\section{References}

Carless, D. \& Douglas, K., 2008. The Role of Sport and Exercise in Recovery from Serious Mental Illness: Two Case Studies. International Journal of Men’s Health, 7(2), pp.137-156.

Cavendish, C., 2013. The Cavendish Review: An Independent Review into Healthcare Assistants and Support Workers in the NHS and social care settings. Department of Health, (July), pp.1-92.

Cleary, M. et al., 2011. Ethnographic Research into Nursing in Acute Adult Mental Health Units: A Review. Issues in Mental Health Nursing, 32(7), pp.424-435. Available at: http://www.tandfonline.com/doi/full/10.3109/01612840.2011.563339.

Crone, D. et al., 2008. Uptake and participation in physical activity referral schemes in the UK: An investigation of patients referred with mental health problems. Issues in Mental Health Nursing, 29(10), pp.1088-1097.

Cullen, C. \& Mccann, E., 2015. Exploring the role of physical activity for people diagnosed with serious mental illness in Ireland. Journal of Psychiatric and Mental Health Nursing, 22(1), pp.58-64.

Day, M. \& Johnson, M., 2017. Working together to address obesity in adult mental health secure units A systematic review of the evidence and a summary of the implications for practice,

Deci, E.L. \& Ryan Richard, M., 2008. Facilitating Optimal Motivation and Psychological Well-Being Across Life’s Domains. Canadian Psychology, 49(1), pp.14-23.

Farholm, A. \& Sørensen, M., 2016. Motivation for physical activity and exercise in severe mental illness: A systematic review of cross-sectional studies. International Journal of Mental Health Nursing, 25(2).

Faulkner, G. \& Biddle, S., 2001. Exercise and mental health: It’s just not psychology! Journal of Sports Sciences, 19(6), pp.433-444. Available at: http://www.tandfonline.com/doi/abs/10.1080/026404101300149384.

Faulkner, G. \& Biddle, S.J.H., 2002. Mental health nursing and the promotion of physical activity. Journal of Psychiatric and Mental Health Nursing, 9(6), pp.659-665. Available at: http://doi.wiley.com/10.1046/j.1365-2850.2002.00520.x.

Happell, B. et al., 2012. Nurses' views on physical activity for people with serious mental illness. Mental Health and Physical Activity, 5(1), pp.4-12. Available at: http://linkinghub.elsevier.com/retrieve/pii/S1755296612000075.

Happell, B., Platania-Phung, C. \& Scott, D., 2011. Placing physical activity in mental health care: A leadership role for mental health nurses. International Journal of Mental Health Nursing, 20(5), pp.310-318. Available at: http://doi.wiley.com/10.1111/j.1447-0349.2010.00732.x.

Kramer, L. V. et al., 2014. Correlates of reduced exercise behaviour in depression: The role of motivational and volitional deficits. Psychology and Health, 29(10), pp.1206-1225. Available at: http://dx.doi.org/10.1080/08870446.2014.918978.

Ng, J.Y.Y. et al., 2012. Self-Determination Theory Applied to Health Contexts: A Meta-Analysis. Perspectives on Psychological Science, 7(4), pp.325-340.

Phillips, K.G., Beretta, A. \& Whitaker, H.A., 2014. Brain, Mind and Consciousness in the History of Neuroscience. , 6, pp.355-369. Available at: http://link.springer.com/10.1007/978-94-017-87741 .

Ribe, A.R. et al., 2014. Long-term mortality of persons with severe mental illness and diabetes: a 
population-based cohort study in Denmark. Psychological Medicine, 44(14), pp.3097-3107.

Robertson, J. et al., 2000. Lifestyle related risk factors for poor health in residential settings for people with intellectual disabilities. Research in Developmental Disabilities, 21(6), pp.469-486. Available at: http://linkinghub.elsevier.com/retrieve/pii/S0891422200000536.

Schuch, F. et al., 2017. Physical activity and sedentary behavior in people with major depressive disorder: A systematic review and meta-analysis. Journal of Affective Disorders, 210(August 2016), pp.139-150. Available at: http://dx.doi.org/10.1016/j.jad.2016.10.050.

Stanton, R. \& Happell, B., 2014. Exercise for mental illness: A systematic review of inpatient studies. International Journal of Mental Health Nursing, 23(3), pp.232-242.

Suija, K. et al., 2009. Physical activiy of depressed patients and their motivation to exercise: nordic Walking in family practice. International Journal of Rehabilitation Research, 32(2), pp.132138.

Vancampfort, D. et al., 2016. Cardiorespiratory Fitness in Severe Mental Illness : A Systematic Review and Meta-analysis. Sports Medicine, 47(2), pp.343-352. Available at: "http://dx.doi.org/10.1007/s40279-016-0574-1.

Vancampfort, D. et al., 2013. The importance of self-determined motivation towards physical activity in patients with schizophrenia. Psychiatry Research, 210(3), pp.812-818. Available at: http://dx.doi.org/10.1016/j.psychres.2013.10.004.

Walker, E.R., McGee, R.E. \& Druss, B.G., 2015. Mortality in Mental Disorders and Global Disease Burden Implications. JAMA Psychiatry, 72(4), pp.334-341. Available at: http://archpsyc.jamanetwork.com/article.aspx?doi=10.1001/jamapsychiatry.2014.2502ไnhttp://w ww.ncbi.nlm.nih.gov/pubmed/25671328.

Western, S. et al., 2014. Physical activity interventions: an essential component in recovery from mental illness. British Journal of Sports Medicine, 0(0), pp.2011-2013. Available at: http://bjsm.bmj.com/cgi/doi/10.1136/bjsports-2014-094314.

Williams, T.L. et al., 2017. Exercise is medicine? Most of the time for most; but not always for all. Qualitative Research in Sport, Exercise and Health, 6778(December), pp.1-16. Available at: https://www.tandfonline.com/doi/full/10.1080/2159676X.2017.1405363. 